\title{
A Study of the Effect of Gold Thickness Distribution in the Jet Plating Process to Optimize Gold Usage and Plating Voltage Using Design of Experiments
}

\author{
Chuckaphun Aramphongphun ${ }^{1, \mathrm{a}}{ }^{*}$ and Chalermpol Nampanya ${ }^{2, \mathrm{~b}}$ \\ ${ }^{1}$ Department of Industrial Engineering, Faculty of Engineering, Kasetsart University, \\ 50 NgamWongWan Road, Chatuchak, Bangkok 10900, Thailand \\ ${ }^{2}$ Graduate Program in Engineering Management, Department of Industrial Engineering, \\ Faculty of Engineering, Kasetsart University, 50 NgamWongWan Road, Chatuchak, \\ Bangkok 10900, Thailand \\ afengchar@ku.ac.th, ${ }^{1}$ bjack_chalermpol@hotmail.com
}

\begin{abstract}
A gold plating process in the electronics industry can be classified as (i) all surface plating or (ii) selective plating. Selective plating is more widely used than all surface plating because it can save more gold used in the plating process and takes less plating time. In this research, the selective plating process called jet plating was studied. Factors that possibly affected the gold usage and plating voltage were also studied to reduce the production cost. These factors included (a) plating temperature, (b) crystal (inhibitor) amount, (c) distance between workpiece and anode, (d) plating current and (e) plating speed. A two-level Full Factorial design with center points was first performed to screen the factors. A Central Composite Design (CCD) was then employed to optimize the factors in jet plating. The amount of gold usage should be reduced to $0.366 \mathrm{~g} / 10,000$ pieces, the plating speed should be increased to $4 \mathrm{~m} / \mathrm{min}$ and the plating voltage should not exceed $8.0 \mathrm{~V}$. According to the analysis, the optimal settings should be as follows: the plating temperature at $55.5 \mathrm{deg} \mathrm{C}$, the crystal amount at $90 \%$, the distance at $0.5 \mathrm{~mm}$, the plating current at $2.8 \mathrm{~A}$, and the plating speed at $4.5 \mathrm{~m} / \mathrm{min}$. This optimal setting led to gold usage of $0.350 \mathrm{~g} / 10,000$ pieces and a plating voltage of $7.16 \mathrm{~V}$. Confirmation runs of 30 experiments at the optimal conditions were then performed. It was found that the gold usage and the plating voltage of the confirmation runs were not different from the optimized gold usage and plating voltage. The optimal condition was then applied in production, which could reduce the gold usage by $4.5 \%$ and increase the plating speed by $12.5 \%$ while the plating voltage did not exceed the limit.
\end{abstract}

\footnotetext{
* Corresponding author: fengchar@ku.ac.th,
} 


\section{Introduction}

In the current situation, electronic devices such as smart phones and tablets play an important role in daily life. Various brands are available and have different features and prices. When a new model of a product is launched onto the market, its price is often lower than the previous model. This lower price of the product could be achieved by reducing component cost in the assembly to increase the opportunity in the competitive market. Preassembled parts manufacturers must therefore find ways to reduce the production cost as much as possible. The cost mainly includes the raw materials cost and operating time cost, which are direct costs in production. If the amount of raw materials used in production can be reduced while performance of the products is maintained, the manufacturer still delivers the same good product to the customers. Moreover, the operating time in production can also be improved to increase productivity. This cost reduction in both raw materials and operating time in production could lead to a significant increase in profit.

One of the processes in manufacturing electronic devices is electro-plating. Metals that are commonly used in the electroplating process are gold, tin, nickel and chromium [1]. This research work mainly focuses on the gold plating process using the injection method also known as jet plating, which is a selective plating process as shown in Fig. 1. In addition, because the metal used in the jet plating process is gold, the manufacturing cost is relatively expensive.

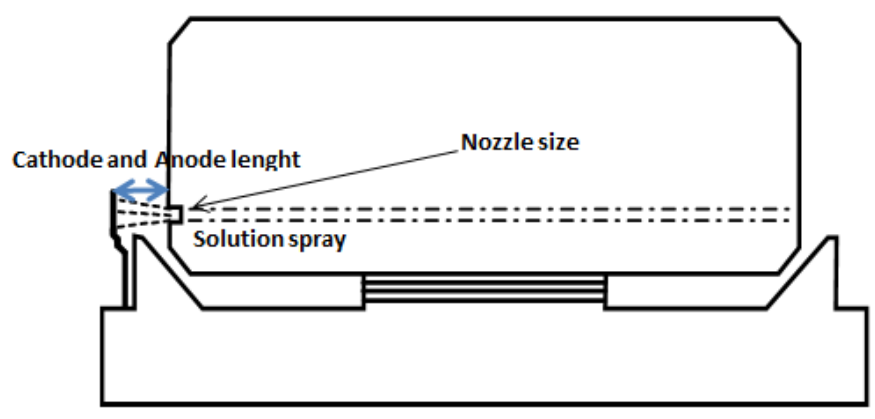

Figure 1. Schematic of the jet plating process

According to Fig. 2, the jet plating process consists of (i) electro-degreasing to clean the workpiece by electricity. This step is used to clean oil residuals and metal chips from the metal forming process. (ii) acid pickling to activate the surface and remove metal oxides before nickel plating. (iii) nickel plating to increase the strength of the workpiece and to prevent rust from the metal layer. (iv) surface activation and cleaning prior to gold plating. Failure to perform this step may cause a peel-off defect due to the unclean surface. (v) gold plating to conduct electricity and signals as well as to increase solderability. (vi) post treatment to coat the surface with chemicals to prevent rust. 


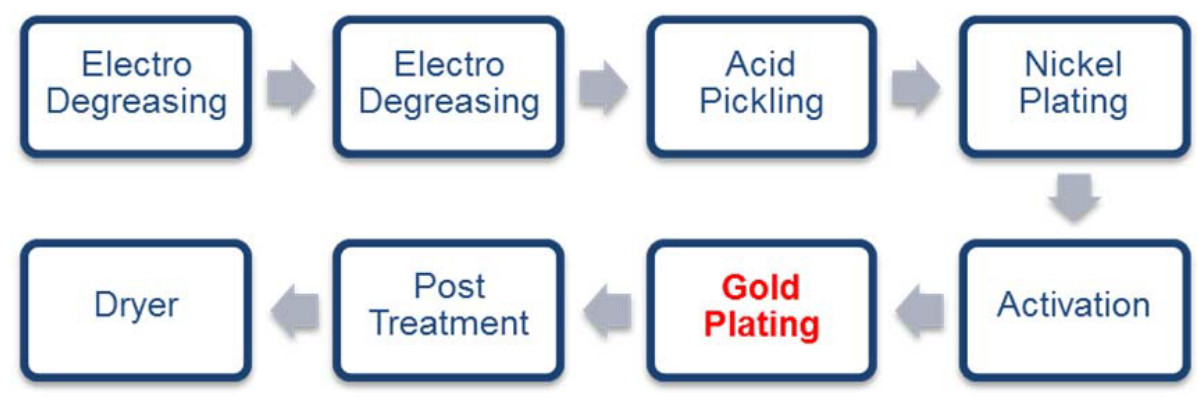

Figure 2. Various steps in the gold plating process

In the electroplating process, the thickness of the coating layer is significantly affected by the plating solution, plating current and time [2-4]. The plating thickness may vary depending on the form of the plating process. In jet plating, the plating solution is sprayed onto the surface where the desired thickness is required $[5,6]$. The plating thickness of the other areas of the workpiece that does not contact the solution should be slightly changed depending on the plating current and time. In addition to the plating solution, plating current and time, distance between the workpiece (cathode) and anode, the injection rate of the solution to the workpiece, and temperature of the plating solution [7] also significantly affect the plating thickness and thickness distribution. Each of these parameters affects the plating thickness differently. The plating current and time as well as the solution concentration are directly proportional to the plating thickness while the temperature of the plating solution is indirectly proportional to the plating thickness depending on the evaporation rate of the plating solution and additives in the plating solution.

This research work mainly focuses on the factors that significantly affect the thickness and the distribution of the gold plating on the workpiece surface $[6,8]$. If the plating process has too great a distribution of the plating solution, this could lead to the waste of the solution in undesired areas. If the distribution of the solution could be minimized to plate only the desired area, this could significantly reduce the raw material cost as well as speed up the plating process. The factors selected in this research included (a) plating temperature, (b) crystal (inhibitor) amount, (c) distance between workpiece (cathode) and anode, (d) plating current, and (e) plating speed. The objectives of this research are (i) to reduce the amount of the gold usage and (ii) to increase the plating speed while the plating thickness of gold on the working surface follows the standard specification.

\section{Materials and Methods}

These five factors as mentioned above were selected to perform the experiments. Two levels (low and high levels or $[-1]$ and $[+1]$ in coded units, respectively) of each factor were applied and summarized as follows: (A) Plating temperature: 50 and 60 degrees Celsius, (B) Crystal or inhibitor amount (Crystal or $\mathrm{Cr}$ agent): $70 \%$ and $90 \%$, (C) Distance between workpiece (cathode) and anode (A-C length): 0.5 and $1.5 \mathrm{~mm}$, (D) Plating current: 2.5 and 2.8 A, and (E) Plating speed: 4.5 and $5.0 \mathrm{~m} / \mathrm{min}$. Responses in this experiment were: (i) amount of gold usage (Au usage) and (ii) plating voltage. The experiment was then designed according to the Design of Experiments (DOE) principle [9]. A two-level Factorial design with center points was used to screen the factors and select the significant factors. The center points were included in the experiment to examine the non-linear 
(curvature) relationships between various factors. If the relationship of factors was nonlinear, Response Surface Methodology (RSM), particularly Central Composite Design (CCD), was then used to design the experiment and to determine the optimal amount of gold usage and plating voltage. The target amount of gold usage should be lower than 0.366 g / 10,000 pieces; the target speed of plating should be greater than $4.0 \mathrm{~m} / \mathrm{min}$, and the voltage should not exceed $8.0 \mathrm{~V}$. The experimental results were then analyzed to determine the optimal parameter setting. Confirmation runs were finally performed at the optimal parameter setting to verify the results.

\section{Results and Discussion}

The experiments were designed according to a two-level Full Factorial design with two replications and six center points. The results of the experiments were then obtained. Analysis of the experimental results was performed to screen the significant factors. The two responses including gold usage and plating voltage were analyzed separately. A residual analysis of these two responses was also performed. It was found that the residuals of the gold usage were independent and normally distributed with constant variance. In addition, it was found that the residuals of the plating voltage were also independent and normally distributed with constant variance. To determine the significant factors and to test the curvature (non-linear) effect, Analysis of Variance (ANOVA) was performed to analyze the experimental results using Minitab version 16. It was found that all five factors significantly affected the gold usage and the curvature testing of the gold usage was significant ( $p$-value $=0.037$ ). In addition, it was found that all five factors significantly affected the plating voltage, and the curvature testing of the plating voltage was also significant ( $\mathrm{p}$-values $<0.000$ ).

According to the previous analyses, it was found that the curvature effects of both the gold usage and plating voltage were significant. Therefore, Response Surface Methodology (RSM) was then used to design the next experiments and to analyze the effect of the second order (non-linear) terms. Because the low and high levels of the factors were selected at the limits of the factors, these levels could not be extended beyond the low and high levels in the previous settings. Therefore, Face-centered Central Composite Design was applied to design the experiments as shown in Table 1. 
TABLE 1. RESULTS OF THE EXPERIMENTS ACCORDING TO FACE-CENTERED CENTRAL COMPOSITE DESIGN

\begin{tabular}{|c|c|c|c|c|c|c|c|}
\hline Run no. & Temp. & Cr agent & A-C length & Current & Speed & Voltage & Au usage \\
\hline 1 & 50 & 70 & 0.5 & 2.50 & 5.00 & 8.8 & 0.377 \\
\hline 2 & 60 & 70 & 0.5 & 2.50 & 5.00 & 9.4 & 0.377 \\
\hline 3 & 50 & 90 & 0.5 & 2.50 & 5.00 & 10.0 & 0.408 \\
\hline 4 & 60 & 90 & 0.5 & 2.50 & 5.00 & 10.6 & 0.400 \\
\hline 5 & 50 & 70 & 1.5 & 2.50 & 5.00 & 7.3 & 0.362 \\
\hline 6 & 60 & 70 & 1.5 & 2.50 & 5.00 & 12.0 & 0.422 \\
\hline 7 & 50 & 90 & 1.5 & 2.50 & 5.00 & 10.0 & 0.420 \\
\hline 8 & 60 & 90 & 1.5 & 2.50 & 5.00 & 8.6 & 0.399 \\
\hline 9 & 50 & 70 & 0.5 & 2.80 & 5.00 & 8.0 & 0.410 \\
\hline 10 & 60 & 70 & 0.5 & 2.80 & 5.00 & 8.5 & 0.388 \\
\hline 11 & 50 & 90 & 0.5 & 2.80 & 5.00 & 8.9 & 0.376 \\
\hline 12 & 60 & 90 & 0.5 & 2.80 & 5.00 & 9.2 & 0.365 \\
\hline 13 & 50 & 70 & 1.5 & 2.80 & 5.00 & 9.9 & 0.400 \\
\hline 14 & 60 & 70 & 1.5 & 2.80 & 5.00 & 10.7 & 0.411 \\
\hline 15 & 50 & 90 & 1.5 & 2.80 & 5.00 & 11.1 & 0.388 \\
\hline 16 & 60 & 90 & 1.5 & 2.80 & 5.00 & 8.0 & 0.358 \\
\hline 17 & 55 & 80 & 1.0 & 2.65 & 4.75 & 8.0 & 0.364 \\
\hline 18 & 55 & 80 & 1.0 & 2.65 & 4.75 & 8.0 & 0.355 \\
\hline 19 & 55 & 80 & 1.0 & 2.65 & 4.75 & 7.9 & 0.365 \\
\hline 20 & 50 & 80 & 1.0 & 2.65 & 4.75 & 8.0 & 0.368 \\
\hline 21 & 60 & 80 & 1.0 & 2.65 & 4.75 & 7.9 & 0.371 \\
\hline 22 & 55 & 70 & 1.0 & 2.65 & 4.75 & 8.0 & 0.378 \\
\hline 23 & 55 & 90 & 1.0 & 2.65 & 4.75 & 8.1 & 0.347 \\
\hline 24 & 55 & 80 & 0.5 & 2.65 & 4.75 & 7.7 & 0.360 \\
\hline 25 & 55 & 80 & 1.5 & 2.65 & 4.75 & 8.1 & 0.349 \\
\hline 26 & 55 & 80 & 1.0 & 2.50 & 4.75 & 7.7 & 0.344 \\
\hline 27 & 55 & 80 & 1.0 & 2.80 & 4.75 & 8.0 & 0.366 \\
\hline 28 & 55 & 80 & 1.0 & 2.65 & 4.50 & 7.8 & 0.368 \\
\hline 29 & 55 & 80 & 1.0 & 2.65 & 5.00 & 7.7 & 0.351 \\
\hline 30 & 55 & 80 & 1.0 & 2.65 & 4.75 & 8.0 & 0.366 \\
\hline 31 & 55 & 80 & 1.0 & 2.65 & 4.75 & 7.9 & 0.370 \\
\hline 32 & 55 & 80 & 1.0 & 2.65 & 4.75 & 8.0 & 0.374 \\
\hline
\end{tabular}

A regression analysis of the CCD experiments was performed to estimate the coefficients of the second-order regression equation. The reduced regression equations of the gold usage and the plating voltage (as shown in Eq. 1 and Eq. 2, respectively) in terms of the significant factors (in coded units: [-1] and [+1] or low and high levels, respectively) were summarized as follows.

The gold usage $\left(\hat{Y}_{1}\right)$ :

$$
\begin{aligned}
\hat{Y}_{1}= & 0.3663-0.002265 * \text { Temp }-0.004118 * \text { Crystal }-0.002603 * \text { Current }+0.005324 * \text { Speed } \\
& +0.012830 * \text { Temp } * \text { Temp }+0.004609 * \text { Current } * \text { Speed } .
\end{aligned}
$$

The plating voltage $\left(\hat{Y}_{2}\right)$ : 


$$
\begin{aligned}
\hat{Y}_{2}= & 8.4309-0.0077 * \text { Temp }-0.0124 * \text { Crystal }+0.4679 * \text { AC length }-0.2177 * \text { Current } \\
& -0.0086^{*} \text { Speed }+1.1918^{*} \text { Temp }{ }^{*} \text { Temp }+1.3168 * \text { Crystal } * \text { Crystal } \\
& -1.7582 * \text { AC length } * \text { AC length }-0.4567 * \text { Temp } * \text { Crystal }-0.3970 * \text { Temp } * \text { AC length } \\
& -0.3255^{*} \text { Crystal } * \text { AC length }+0.3501 * \text { Current } * \text { Speed. }
\end{aligned}
$$

By minimizing both the gold usage and the plating voltage using the desirability function, the optimal values of the responses and the corresponding values of the factors

\begin{tabular}{|c|c|c|c|c|c|}
\hline $\begin{array}{cc}\text { Optimal } & \\
\text { D } & \text { High } \\
0.68394 & \text { Cur } \\
0.68 & \text { Low }\end{array}$ & $\begin{array}{c}\text { Temp } \\
60.0 \\
{[55.4545]} \\
50.0\end{array}$ & $\begin{array}{c}\text { Crystal } \\
90.0 \\
{[89.5960]} \\
70.0\end{array}$ & $\begin{array}{c}\text { Anode } \\
1.50 \\
{[0.50]} \\
0.50\end{array}$ & $\begin{array}{c}\text { Current } \\
2.80 \\
{[2.80]} \\
2.50\end{array}$ & $\begin{array}{c}\text { Speed } \\
5.0 \\
{[4.50]} \\
4.50\end{array}$ \\
\hline $\begin{array}{c}\text { Composite } \\
\text { Desirability } \\
0.68394\end{array}$ & & & & & \\
\hline $\begin{array}{c}\text { Voltage } \\
\text { Minimum } \\
y=7.1640 \\
d=1.0000\end{array}$ & & & & & \\
\hline $\begin{array}{c}\text { Au usage } \\
\text { Minimum } \\
y=0.3497 \\
d=0.46778\end{array}$ & & & & & \\
\hline
\end{tabular}
were obtained as shown in Fig. 3.

Figure 3. The optimization plot used to determine the optimal values of the gold usage and plating voltage

According to the results of the optimization, the optimal settings should be as follows: the plating temperature at $55.5 \mathrm{deg} \mathrm{C}$, the crystal amount at $90 \%$, the distance between the workpiece (cathode) and anode at $0.5 \mathrm{~mm}$, the plating current at $2.8 \mathrm{~A}$, and the plating speed at $4.5 \mathrm{~m} / \mathrm{min}$. This optimal settings led to gold usage of $0.350 \mathrm{~g} / 10,000$ pieces and a plating voltage of $7.16 \mathrm{~V}$. Confirmation runs of 30 experiments at the optimal conditions were then performed and analyzed using t-test. It was found that a $95 \%$ confidence interval of the gold usage was $0.345-0.350 \mathrm{~g} / 1000$ pieces while a $95 \%$ confidence interval of the plating voltage was 7.156-7.230 V. Therefore, the gold usage and the plating voltage of the confirmation runs were not different from the optimized gold usage and plating voltage.

\section{Summary}

This research aims to study the gold thickness and distribution in the jet plating process. A Full Factorial design with center points was first applied to determine the significant factors affecting the gold usage and plating voltage. It was found that all five factors, including (A) the plating temperature, (B) the crystal or inhibitor amount, (C) the distance 
between the workpiece (cathode) and anode, (D) the plating current and (E) the plating speed, were significant. Moreover, non-linear effects of the factors were also significant. The Response Surface Methodology (RSM), in particular Central Composite Design (CCD) was then used to design the experiments. Regression analysis was used to analyze the CCD experiments and to determine the coefficients of the second order regression equations. These regression equations were employed to optimize the gold usage and plating voltage. The corresponding values of the factors were also obtained. The optimal settings could lead to a reduction in gold usage of $5 \%$ and an increase in the plating speed of $12.5 \%$. The results from this research could greatly reduce the raw materials cost and operating time cost in production resulting in more profit for the manufacturer.

\section{References}

1. O. Yutaka and H. Masao, Some recent topics in gold plating for electronics applications, Gold Bulletin, 31 (1998) 3-13.

2. M. Bayramoglua, B. Onatb and N. Gerena, Statistical optimization of process parameters to obtain maximum thickness and brightness in chromium plating, Journal of Materials Processing Technology, 203 (2008) 277-286.

3. A.R. Chowdhury and G.K. Prasad, Reduction of gold-plating thickness variation, Quality Engineering, 11 (1999) 61-67.

4. B. Zhang, W. Wu, S. Yin, S. Li, Y. Luo and X. Bian, Process optimization of electroless copper plating and its influence on electrochemical properties of AB5-type hydrogen storage alloy, Journal of Rare Earth, 28 (2010) 922-926.

5. I.K. Hong, H. Kim and S.B. Lee, Optimization of barrel plating process for electroless Ni-P plating, Journal of Industrial and Engineering Chemistry, 20 (2014) 3767-3774.

6. C.J. Raub, H.R. Ihan and J. Lendvay, Temperature-sensitive properties of gold and gold alloy electrodeposits, Gold Bulletin, 9 (1976) 1213-1228.

7. C. Chandrasekar and S. Pushpavanam, Pulse and pulse reverse plating: conceptual, advantages and applications, Electrochimica Acta, 53 (2008) 3313-3322.

8. V. Marc De and V. Sommer, High-speed plating for electronic applications, Electrochimica Acta, 47 (2002) 106-116.

9. F. Sánchez and J. Vilán, The use of design of experiments to improve a neural network model in order to predict the thickness of the chromium layer in a hard chromium plating process, Mathematical and Computer Modelling, 52 (2010) 11691176 . 